\title{
Modes of Action for Mucosal Vaccine Adjuvants
}

\author{
Taiki Aoshi
}

\begin{abstract}
Vaccine adjuvants induce innate immune responses and the addition of adjuvants to the vaccine helps to induce protective immunity in the host. Vaccines utilizing live attenuated or killed whole pathogens usually contain endogenous adjuvants, such as bacterial cell wall products and their genomic nucleic acids, which act as pathogen-associated molecular patterns and are sufficient to induce adaptive immune responses. However, purified protein- or antigen-based vaccines, including component or recombinant vaccines, usually lose these endogenous innate immune stimulators, so the addition of an exogenous adjuvant is essential for the success of these vaccine types. Although this adjuvant requirement is mostly the same for parental and mucosal vaccines, the development of mucosal vaccine adjuvants requires the specialized consideration of adapting the adjuvants to characteristic mucosal conditions. This review provides a brief overview of mucosa-associated immune response induction processes, such as antigen uptake and dendritic cell subset-dependent antigen presentation. It also highlights several mucosal vaccine adjuvants from recent reports, particularly focusing on their modes of action.
\end{abstract}

Keywords: adjuvant, dendritic cells, vaccine, mucosal adjuvant

\section{Introduction}

$\mathbf{V}$ ACCINATION IS ONE of the most influential achievements in medical history (47). Global smallpox eradication highlights the significant impact of vaccination. The smallpox vaccine was a live attenuated vaccine, and many current vaccines, such as measles, mumps, and rubella, apply the same strategy of utilizing live attenuated organisms for vaccination. Although these live attenuated vaccines are very effective, their application is limited to only immune healthy people due to the potential for live vaccine-derived infection progression in an immunocompromised host. This is a serious disadvantage of live attenuated vaccines, as demonstrated by the recently approved, live attenuated seasonal influenza virus vaccine FluMist $(7,9)$, which is only approved for use in individuals of 2-49 years of age, leaving the most vulnerable populations, such as infants and the elderly, ineligible for FluMist application.

Inactivated or killed vaccines, and particularly recombinant or component vaccines composed of highly purified pathogen-derived antigen, are applicable for use in broader populations because these vaccines are not infectious. However, their immunogenicity, especially in the case of recombinant vaccines, is usually weaker than that of live attenuated vaccines because they lack the endogenous innate immune stimulators that are required for inducing adaptive immune responses to the antigen (26). As such, in many cases, the addition of an external adjuvant as an exogenous innate immune stimulator is necessary to compensate this limitation of the purified vaccine. As examples, MF59 and AS03 were introduced as vaccine adjuvants for the 2009 pandemic influenza vaccine $(42,58)$. Importantly, AS03 adjuvanted influenza vaccine was associated with an abrupt increase of narcolepsy in Finland and Sweden, but not with MF59, and the underlying mechanisms are still not understood $(42,44)$. Given the importance of the production of safe and effective vaccines, this external adjuvant requirement has generated an increased focus on the vaccine adjuvant development $(10,46)$.

There has also been a notable focus on the mucosal route of vaccination in recent years $(30,40,59,60)$. Many pathogenic infections occur at the mucosal surface, making this site the first line of defense. Therefore, inducing protective immune responses by administering vaccines directly at the mucosa is considered an ideal way. However, attempts to develop mucosal vaccines have not yet been successful $(28,39)$. This is partly due to the lack of knowledge of how mucosal adjuvants work and of how to select appropriate (safe and effective) adjuvant candidates for the target disease. Although there are many similarities to the process of adjuvant development for

Vaccine Dynamics Project, BIKEN Innovative Vaccine Research Alliance Laboratories, Research Institute for Microbial Disease, Osaka University, Osaka, Japan.

(C) Taiki Aoshi, 2017; Published by Mary Ann Liebert, Inc. This Open Access article is distributed under the terms of the Creative Commons Attribution Noncommercial License (http://creativecommons.org/licenses/by-nc/4.0/) which permits any noncommercial use, distribution, and reproduction in any medium, provided the original author(s) and the source are credited. 
parental vaccines, the development of mucosal adjuvants requires some specialized considerations. This review provides an overview of vaccine adjuvants and the role of dendritic cell (DC) subsets, after which it highlights several mucosal vaccine adjuvants, especially nasal vaccine adjuvants from recent reports, focusing on their modes of action.

\section{Vaccine Adjuvants and Their Classification}

A variety of substances, including particles, chemicals, and oils, have been demonstrated to work as vaccine adjuvants in animal experiments and clinical trials $(10,46)$. Their modes of action can be categorized into two major classes: innate immune receptor agonists and others (Fig. 1). Innate immune receptor agonists function as pathogen-associated molecular patterns (PAMPs). They are directly recognized by innate immune receptors, such as Toll-like receptors (TLRs), RIG-I-like receptors (RLRs), Nod-like receptors (NLRs), C-type lectin receptors, AIM2-like receptors (ALRs), and cGAS/STING, and induce proinflammatory cytokine and interferon responses, collectively referred to as "innate immune responses" $(5,54,61)$. The modes of action in the "other" category can be further subdivided into damage-associated molecular pattern (DAMP) inducers and pure antigen delivery systems. Adjuvants that are categorized as DAMP inducers do not cause any innate immune responses in vitro (such as cytokine secretion or DC maturation); they only induce innate immune responses in vivo. These injected substances act by damaging the host cells, causing them to release several factors classified as DAMPs (e.g., DNA and RNA), which subsequently activate innate immune receptors $(20,45)$. Notably, DAMP-inducer adjuvants are usually nonpathogen-derived substances, such as lipids, mineral salts, or polymer particles. In contrast, the pure delivery system adjuvants are totally inert both in vitro and in vivo, in terms of innate immune response induction. Instead, they very efficiently deliver the vaccine antigen to antigen-presenting cells, such as DCs, and the subsequent DC-mediated antigen presentation results in an enhanced adaptive immune response (Fig. 1).

\section{Antigen Uptake Through Mucosal Surfaces}

Mucosal surfaces, which by definition are covered by mucus, act as physical barriers preventing vaccine antigens and adjuvants from reaching the mucosal epithelial cells and other potential antigen transporter cells, such as goblet cells (36), transepithelial dendrite (TED)-forming CX3CR $1^{+}$ macrophages (35), M cells (25), and intraepithelial DCs $(17,64)$ at the mucosa. The cells composing the mucosal epithelium are mutually interconnected by tight junctions and form an impermeable barrier to foreign substances (65). Small chemicals, such the c-di-GMP adjuvant (discussed below; molecular weight $=\sim 690 \mathrm{~g} / \mathrm{mol}$ ), can be passively diffused and are able to cross this barrier through the

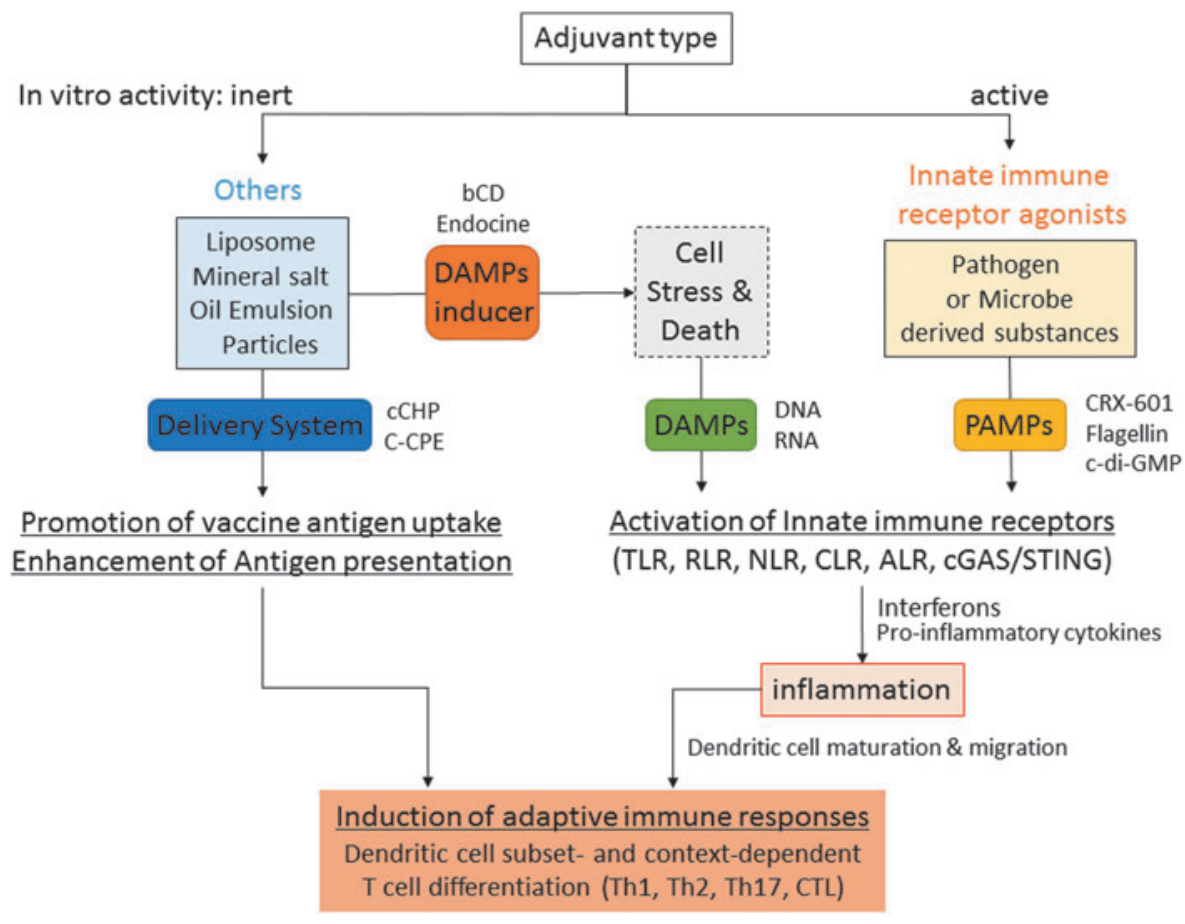

FIG. 1. Adjuvant classification chart based on the mode of action. The adjuvant can be divided into two types based on the presence or absence of in vitro innate immune stimulating activities. "Innate immune receptor agonists" are mostly pathogen- or microbe-derived substances, and work as PAMPs. "Others" mainly consist of nonpathogen-derived substances. "Others" are further divided into "DAMP inducer" and "Delivery system." Both PAMP and DAMP adjuvants activate innate immune receptors and resulted in cytokine responses and dendritic cell maturation/migration. "Delivery system" promotes vaccine uptake and enhances antigen presentation by dendritic cells. ALR, AIM2-like receptor; bCD, hydroxypropyl- $\beta$-cyclodextrin; cCHP, cationic cholesteryl group-bearing pullulan; C-CPE, a C-terminal fragment of Clostridium perfringens enterotoxin; CLR, C-type lectin receptor; DAMP, damage-associated molecular pattern; NLR, Nod-like receptor; PAMP, pathogen-associated molecular pattern; RLR, RIG-I like receptor, TLR, Toll-like receptor. 


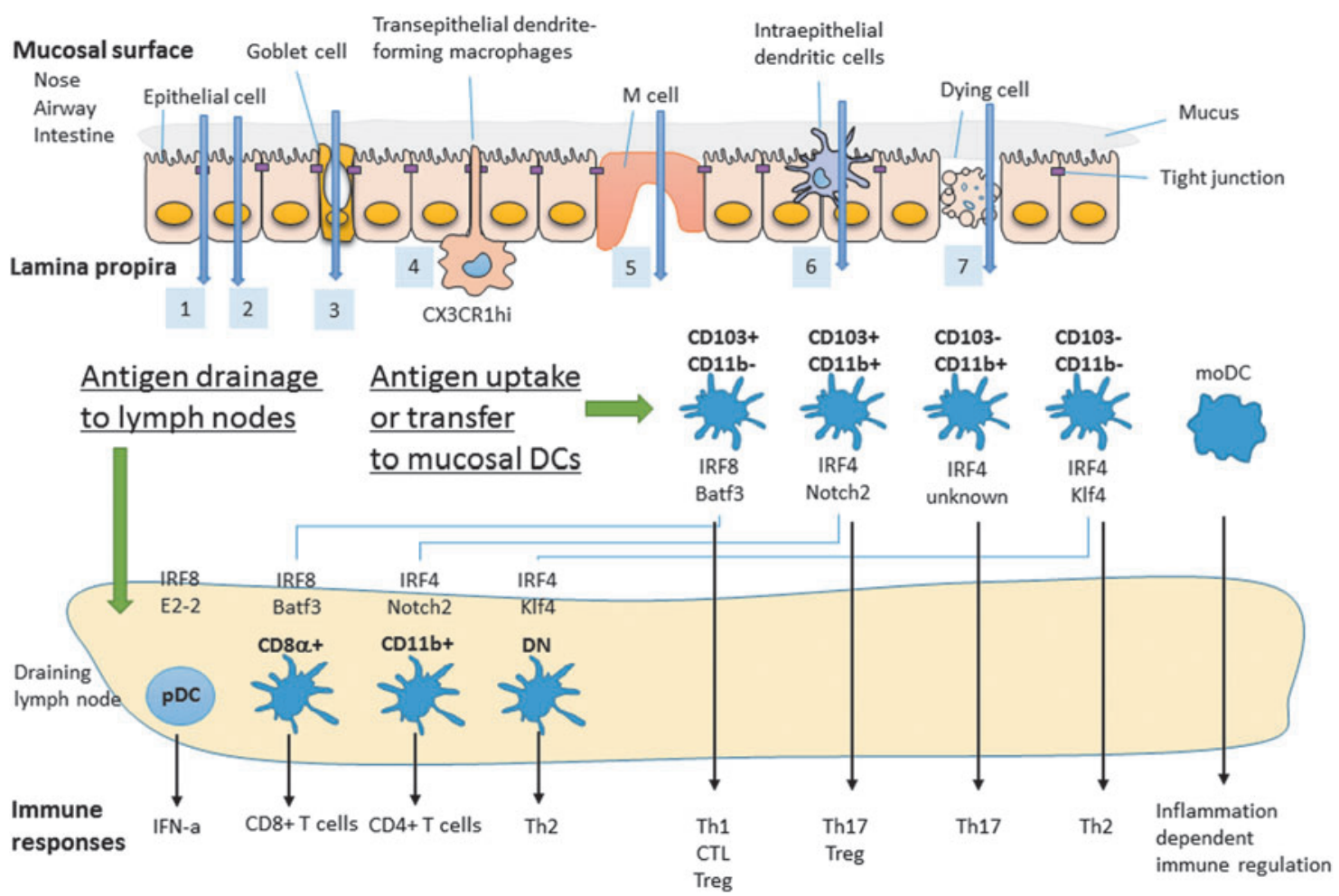

FIG. 2. Antigen transport across the mucosal barrier and DC subset-dependent immune responses. Antigen and adjuvant can cross the mucosal barrier through the following pathways: (1) paracellular pathway, (2) transepithelial pathway, (3) goblet pathway, (4) TED pathway, (5) M-cell pathway, (6) IED pathway, and (7) epithelial cell damage pathway. The translocated antigens and adjuvants are subsequently taken up by several different mucosal tissue DCs. These antigencarrying DCs migrate to the draining lymph nodes for antigen presentation to $\mathrm{T}$ cells, where they induce characteristic $\mathrm{T}$ cell differentiation dependent on their specialized function and associated immune context. IED, intraepithelial dendritic cell; TED, transepithelial dendrite.

intercellular space between epithelial cells; this mechanism is called the paracellular pathway (Fig. 2, pathway 1). Epithelial cell-targeted antigens, such as FcRn- (63) or claudin 4- (53) targeted antigens (both discussed below), are transported by receptor-mediated transcytosis of epithelial cells in the transepithelial pathway (Fig. 2, pathway 2). Experimental soluble antigens, such as ovalbumin or dextran, can be taken up by goblet cells in the goblet pathway (Fig. 2, pathway 3) (36) or by TED-forming CX3CR $1^{+}$macrophages in the TED pathway (Fig. 2, pathway 4) (35). Nanoparticles and some bacteria are taken up by $\mathrm{M}$ cells in the $\mathrm{M}$-cell pathway (25) (Fig. 2, pathway 5) or by intraepithelial DCs in the intraepithelial DC (IED) pathway $(17,64)$ (Fig. 2, pathway 6). Epithelial cell damage also physically breaks the mucosal barrier, allowing antigens to be transported into the lamina propria through the epithelial cell damage pathway (Fig. 2, pathway 7). For all pathways, the translocated antigens are taken up by mucosal tissue DCs, after which some of these antigen-carrying DCs migrate to the draining lymph nodes.

\section{Subsets in Lymphoid and Mucosal Tissues}

DCs are important cells that bridge innate and adaptive immune responses, and they have been shown to play a critical role in antigen presentation and tolerance induction. Recent extensive analyses of the cell surface makers and the critical growth and transcription factors involved in DC dif- ferentiation have established that DCs form a heterogeneous cell population, and their comprehensive transcriptome data are also open for public use by the Immunological Genome Project (www.immgen.org). These DC subsets are functionally distinct and differentially affect $\mathrm{T}$ cell differentiation into Th1, Th2, Th17, CTL, and regulatory T (Treg) cells $(21,37,38,50)$. Although most of the experiments defining these subsets were performed in mice, similar DC subsets have been shown to exist in humans (49). In steady-state lymphoid tissue, conventional DCs (cDCs) and plasmacytoid DCs (pDCs) are identified residentially. Lymphoid tissueresident $\mathrm{cDCs}$ are further divided to three $\mathrm{cDC}$ subsets: $\mathrm{CD} 8 \alpha^{+} / \mathrm{CD}^{-} / \mathrm{CD} 11 \mathrm{~b}^{-} / \mathrm{CD} 24^{+} / \mathrm{SIRP} \alpha^{-}$(referred to in this review as $\left.\mathrm{CD} 8 \alpha^{+}\right) \mathrm{DC}, \mathrm{CD} 8 \alpha^{-} / \mathrm{CD} 4^{+} / \mathrm{CD} 11 \mathrm{~b}^{+} / \mathrm{CD} 24^{-} / \mathrm{SIRP} \alpha^{+} /$ $\mathrm{ESAM}^{\mathrm{hi}}\left(\mathrm{CD} 11 \mathrm{~b}^{+}\right) \mathrm{DC}$, and $\mathrm{CD} 8 \alpha^{-} / \mathrm{CD} 4^{-} / \mathrm{CD} 11 \mathrm{~b}^{+} / \mathrm{CD} 24^{-} /$ $\mathrm{SIRP} \alpha^{-} / \mathrm{ESAM}^{\mathrm{lo}}$ double-negative (DN) DC $(2,37,38)$ (Fig. 2). In peripheral nonlymphoid tissue, such as the lung and intestinal lamina propria, four phenotypically different DC

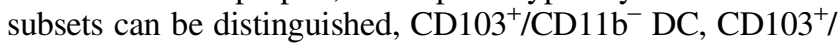

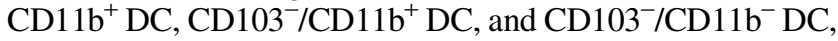
but these occur in different population ratios depending on the examined organ $(8,16,51)$ (Fig. 2, bottom). Analyses from several transcriptional factor-deficient mice revealed that $\mathrm{pDC}$, $\mathrm{CD} 8 \alpha^{+} \mathrm{DC}$, and $\mathrm{CD}_{103}{ }^{+} / \mathrm{CD} 11 \mathrm{~b}^{-} \mathrm{DC}$ are an IRF8-dependent

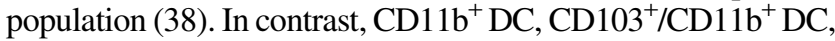

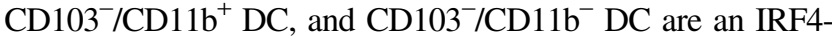
dependent population $(38,51,55)$. An analysis using Batf3- 
deficient mice (24) showed that lymphoid tissue-resident $\mathrm{CD} 8 \alpha^{+}$DCs and peripheral tissue $\mathrm{CD} 103^{+} / \mathrm{CD} 11 \mathrm{~b}^{-}$DCs are related populations (16). Similarly, Notch2 is required for lymphoid tissue $\mathrm{CD}_{11 b^{+}}$DCs and peripheral tissue $\mathrm{CD}_{103}{ }^{+} /$

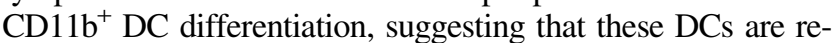
lated populations $(29,48)$. Furthermore, Klf4 is required for DN DCs and CD103- ${ }^{-}$CD11b ${ }^{-}$DCs (55), suggesting that these two DC subsets are also related through Klf4 dependency.

These lymphoid tissue-resident and peripheral nonlymphoid/migratory DCs have largely distinct, but some overlapping, functions. Seminal work by Dudziak et al. first demonstrated that lymphoid tissue-resident CD8 $\alpha^{+}$DCs and $\mathrm{CD} 11 \mathrm{~b}^{+} \mathrm{DCs}$ are functionally different for preferential antigen presentation to $\mathrm{CD}^{+} \mathrm{T}$ cells and preferential antigen presentation to $\mathrm{CD} 4^{+} \mathrm{T}$ cells, respectively (15) (Fig. 2). This DC subset marker and the functional connection seem to also hold true for other lymphoid and nonlymphoid DC

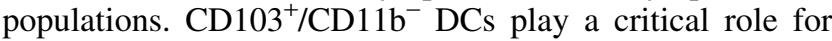
Th1 cell $(34)$ and CTL $(14,16,19,24)$ induction and are also involved in some part of Treg induction (11). In addition, $\mathrm{CD}_{103}{ }^{+} / \mathrm{CD}_{11 \mathrm{~b}^{+}}$DCs are involved in Th17 and Treg cell

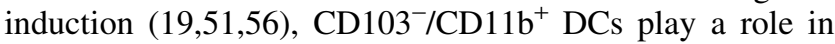
Th17 cell induction $(8,51)$, and $\mathrm{CD} 103^{-} / \mathrm{CD} 11 \mathrm{~b}^{-}$DCs are a factor in Th2 cell induction (55).

\section{Adjuvant Sensing at the Mucosal Surface}

Many cell surface and endosome innate immune receptors are characteristically expressed on limited cell types. For example, TLR3 is specifically expressed on CD8 $\alpha^{+}$DCs (12). In contrast, most cytosolic innate immune receptors, such as RLR, NLR, ALR, and cGAS/STING, are thought to be ubiquitously expressed by a variety of cell types. In addition to their basal expression, the expression levels of some innate immune receptors are upregulated by inflammatory situations (6). The mucosal epithelium expresses many innate immune receptors, including TLRs, and a variety of TLR agonists have been reported to work as mucosal adjuvants, including Muramyldipeptide, PolyIC, MPL, flagellin, and $\mathrm{CpG}$ $(1,30,59,60)$. However, which TLRs are expressed in the mucosal epithelium is sometimes controversial and difficult to determine because epithelium sample preparation can easily become contaminated with other immune cells, such as macrophages and DCs, which can express various TLRs (1). In addition, many bacterial toxins, including cholera toxin and heat-labile enterotoxin, are well-known experimental mucosal adjuvants without being TLR agonists, but their mode of action is not yet well understood.

Furthermore, as mentioned above, nonmicrobial-derived products, including liposomes, oil emulsions, and several kinds of nanoparticles, also act as mucosal adjuvants. Some of these products function as a true delivery system, while others induce local and temporal mucosal epithelial damage or stress, which stimulates the release of DAMPs. The released DAMPs are then recognized by other epithelial cells and mucosal immune cells, including DCs, resulting in innate immune responses. It is likely that many mucosal adjuvants directly act on both epithelial and mucosal DCs, such that both contribute to the adjuvant effect. As discussed further below, flagellin works directly on the TLRexpressing epithelium, but TLR5 expression on the mucosal DC is not required for the adjuvanticity of flagellin in the nasal route $(18,57)$, indicating that some of the effects of this mucosal adjuvant on mucosal DCs are indirect.

\section{Mucosal Adjuvants and Their Modes of Action}

A number of parental vaccine adjuvants also work as mucosal adjuvants, and many have been tested in animals via the intranasal route. In this study, some representative intranasal mucosal adjuvants are highlighted; they are categorized as (1) direct innate immune receptor agonist acting as PAMPs, (2) DAMP inducer, or (3) delivery systems, based on their apparent modes of action (Fig. 1).

\section{PAMP adjuvants}

Many microbe-derived substances, including TLR ligands, work as mucosal adjuvants through their specific innate immune receptor-mediated signaling in the host cells.

Bis- $\left(3^{\prime}, 5^{\prime}\right)$-cyclic dimeric guanosine monophosphate (c-diGMP) is a bacterial intracellular signaling molecule that has been reported as a potent mucosal vaccine adjuvant for inducing Th1 and Th17 immune responses for a plant-derived H5 influenza vaccine (31). C-di-GMP is known as an agonistic ligand of STING, which recognizes cytosolic cyclic dinucleotides and activates the TBK1-IRF3 axis of IFN-I responses. Although the adjuvanticity of c-di-GMP as a mucosal adjuvant was completely lost in STING-deficient mice, the adjuvant effect of c-di-GMP is independent of IFN-I signaling and is dependent on the STING-NFKB axis of TNF $\alpha$ signaling in DCs (3). Blaauboer et al. further demonstrated that c-di-GMP, which is not cell membrane permeable, is taken up by pinocytosis-efficient DCs after nasal administration in vivo and stimulates antigen uptake in DCs and epithelial cells (4). They also found that c-di-GMP treatment in vivo can activate STING-independent signaling in cells other than DCs, resulting in IL-1, IL-6, IL-33, and TSLP secretion, however, the details of this c-di-GMP-mediated STING-independent IL- $1 \alpha$, IL-33, and TSLP secretion were not known (4). These data suggest that c-di-GMP works as a potent mucosal adjuvant by enhancing the whole process involved in DC-mediated antigen presentation to $\mathrm{T}$ cells, from the mucosal surface to the draining lymph nodes.

The TLR5 agonist flagellin is an effective mucosal vaccine adjuvant. TLR5 is specifically expressed on the subset

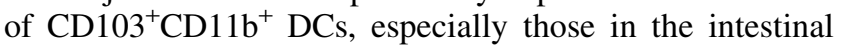
lamina propria, and TLR5 signaling in this DC subset has been shown to play an important role in intestinal IgA production and Th17 differentiation (56). However, the mode of action of flagellin in the airway mucosa (which

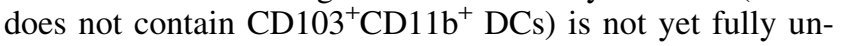
derstood. Recent reports demonstrated that the mucosal adjuvant activity of flagellin does not require TLR5expressing hematopoietic cells, including DCs. Instead, flagellin acts on the airway epithelial cells that express TLR5 (18,57). These studies suggest that flagellin first activates the airway mucosal epithelial cells and then indirectly activates the airway resident cDCs, which are essential to the flagellin mucosal adjuvant activity.

\section{DAMP adjuvants}

Innate immune receptors also recognize self-derived substances that are released or secreted on cellular stress or 
death. Recent studies have suggested that some adjuvants act as DAMP adjuvants in the nasal mucosa.

Hydroxypropyl- $\beta$-cyclodextrin (bCD) is a commonly used excipient to solubilize pharmaceutical agents of poor solubility in water. Recent studies demonstrated that bCD works as a vaccine adjuvant when it is administered with vaccine antigen via a subcutaneous (43) or intranasal route (27). A local and temporal high concentration of bCD at the site of administration causes local cellular stress and death, resulting in host cell DNA release. This released DNA works as a DAMP to induce Th2 immune responses in a TBK1 signaling-dependent manner.

Endocine $^{\mathrm{TM}}$ is a lipid-based mucosal adjuvant consisting of oleic acid and mono-olein, and it is under development as a nasal influenza vaccine adjuvant (32) for clinical use, however, the mechanisms of action were not known. A recent study demonstrated that although Endocine is a lipid adjuvant, neither TLR2 nor TLR4, either of which may recognize a lipid moiety in the adjuvant, is required for the adjuvanticity of Endocine in a mouse model. In an in vitro DC stimulation study, Endocine rapidly killed cultured DCs in a concentration-dependent manner. After intranasal administration, Endocine induced local cell death, which was associated with lactate dehydrogenase, DNA, and RNA release in the nasal wash (these are indicating cell death at the nasal mucosa, including many different cell types). Similar to bCD, the adjuvant activity of Endocine was dependent on a TBK1 signaling pathway, suggesting the involvement of DAMPs. Coadministration of RNase A, but not of DNase I, significantly reduced the Endocinemediated antibody response; however, neither treatment reduced the cholera toxin-mediated antibody response, suggesting that the adjuvanticity of Endocine is characteristically mediated by host cell-released RNA as a DAMP (23).

\section{Delivery system adjuvants}

It also has been shown that enhancing nasal mucosal surface attachment by using cationic nanometer-sized gels or by applying bioengineered targeting of epithelial cell surface molecules is effective for augmenting mucosal immune responses, and, in many cases, these approaches seem not to require additional PAMP/DAMP adjuvants.

Cholesteryl group-bearing pullulan (CHP) is a selfassembled polymer-based nanoparticle carrier used to deliver drugs or vaccine antigens. Nochi et al. demonstrated that with botulinum antigen encapsulation, which they called CHP-BoHc/A, the CHP-BoHc/A did not show any improvement compared with BoHc/A alone. However, cationic $\mathrm{CHP}$ with BoHc/A (cCHP-BoHc/A) showed significant induction of antigen-specific local and systemic IgA and $\mathrm{IgG}$ responses. This improvement was associated with prolonged $(\sim 10 \mathrm{~h})$ retention of cCHP-BoHc/A in the nasal cavity. Epithelial cell-attached cCHP-BoHc/A was internalized by both epithelial cells and $\mathrm{M}$ cells, and the antigen was subsequently taken up by $\mathrm{CD} 11 \mathrm{c}^{+}$DCs. Interestingly, increased DC maturation was not observed in either in vitro or in vivo experiments. The authors concluded that effective antigen delivery through the mucosa via cCHP is sufficient for the induction of mucosal immune responses (41).
FcRn is a receptor of $\operatorname{IgG}$ that allows fetuses to obtain maternal $\operatorname{IgG}$ through the placental and intestinal routes, and it has been observed that FcRn can transport $\operatorname{IgG}$ across mucosal epithelia in adults. Ye et al. reported that intranasal immunization using HSV-2 glycoprotein $\mathrm{gD}$ fused with the $\mathrm{IgG} \mathrm{Fc}$ fragment acts as a protective mucosal vaccine against intravaginal virulent HSV-2 186 strain challenge. They demonstrated that this was mediated by FcRn using gD fused with mutant Fc, which lacked FcRn binding, or with FnRn knockout mice. The authors concluded that Fc-fused antigens are efficiently transported by nasal epithelial cells in an FcRndependent manner and are subsequently transferred to mucosal antigen-presenting cells, such as DCs (63).

The C-terminal fragment of Clostridium perfringens enterotoxin (C-CPE) has a high binding affinity for nasal epithelial cells expressing claudin-4. Bioengineering of a vaccine antigen fused with $\mathrm{C}-\mathrm{CPE}$ works as another vaccine antigen targeting method for the nasal mucosal epithelium. Suzuki et al. demonstrated that pneumococcal surface protein A (PspA) fused with C-CPE induced protective systemic and respiratory antibody responses in mice, which are associated with PspA-C-CPE binding to the nasal epithelium and $\mathrm{M}$ cells (53).

\section{Context-Dependent Mucosal Immune Responses}

Although each DC subset has specialized default functions, several recent reports have suggested that its functions are also regulated by the associated immune context. DePaolo et al. demonstrated that retinoic acid, which is reported to play a critical role in Treg differentiation through $\mathrm{CD}_{103^{+}}$DCs in the intestine, also acts as an adjuvant for inducing $\mathrm{CD} 4^{+} \mathrm{T}$ cell-mediated autoimmunity against fed antigens when IL-15 is present (13). These findings suggest that the presence of IL-15 regulates tolerance or immunity. In addition, Yang et al. showed that colonization with segmented filamentous bacteria (SFB) induced a Th17 response to the antigen derived from SFB; in contrast, colonization with Listeria monocytogenes expressing this SFB-derived antigen, elicited Th1 instead of Th17 responses (62). It has been known that L. monocytogenes infection typically induces Th1 responses, suggesting that the bacterial host, rather than antigen itself, affects Th differentiation. More recently, Stary et al. used a vaginal mucosal vaccine model for Chlamydia trachomatis (Ct) infection in mice and demonstrated that ultraviolet (UV) light-inactivated $\mathrm{Ct}$ (UV-Ct) generated Tregs via $\mathrm{CD}_{103^{+}}$DC-mediated antigen presentation resulting in nonprotective vaccination, whereas vaccination with UVCt plus R848 containing a PLGA particle adjuvant, which the authors called charge-switching synthetic adjuvant particles (cSAP), activated Ct-specific protective $\mathrm{CD}^{+}{ }^{+} \mathrm{T}$ cell responses via $\mathrm{CD} 103^{-}$DCs. This protective immunity was similar to that induced by vaccination with live $\mathrm{Ct}$ at the vaginal mucosa (52), suggesting that the UV-Ctassociated innate immune signature promotes Tregs, while the cSAP adjuvant promotes effector $\mathrm{CD}^{+} \mathrm{T}$ cells. These reports strongly suggest that antigen presentation by mucosal DC subsets is profoundly influenced by aspects of the surrounding immune environment, such as the presence of particular cytokines, the antigen providing platform, and the specific adjuvant. 


\section{Importance of Selecting an Appropriate Adjuvant and Drug Delivery System}

The development of vaccine antigen purification/production techniques and drug delivery systems has enabled a variety of combinations of antigen and adjuvant, including totally artificial antigen and adjuvant combinations. Maroof et al. reported an insightful example for a split influenza vaccine with a synthetic TLR4 agonist, CRX-601, as an exogenous synthetic adjuvant. Usually, live influenza virus infection and whole inactivated influenza vaccine induce Th1 responses, whereas immunization with this split vaccine induced Th2 responses, and these influenza virus-specific Th1 and $\mathrm{Th} 2$ responses are both protective against influenza virus infection $(22,26)$. Interestingly, intranasal, but not subcutaneous, split vaccine immunization with the CRX-601 adjuvant promoted influenza-specific Th17 responses, and these immunization route- and adjuvant-dependent Th17 responses are detrimental for influenza virus challenge through an IL17A-mediated augmentation of neutrophilic lung inflammation (33). This indicates that at least when considering a mucosal vaccination, the use of Th17-inducing adjuvants should be avoided for influenza split antigen vaccines. Furthermore, the selection of both an appropriate adjuvant and administration route (or drug delivery system) is important for making an effective mucosal vaccine.

\section{Conclusion}

A variety of substances can act as mucosal adjuvants; however, their modes of action are very different. Some directly act on specialized DC subsets, while others work indirectly. Nonmicrobial-derived substances can also function as mucosal adjuvants. Some of these substances act purely as a delivery system; others are DAMP inducers, which indirectly induce innate immune responses. This DAMP inducermediated epithelial cell damage may also break up the mucosal barrier that otherwise prevents vaccine antigen and adjuvant from reaching the mucosal lamina propria where important DC subsets reside. Which types of adjuvant are appropriate is dependent on the target disease, population, and the selected antigen. In some cases, the addition of an adjuvant that induces the same Th type as the original disease antigen is optimal, but in other instances, it may be better to use an adjuvant that induces a different Th type. The selection of adjuvants must aim to appropriately balance safety and efficacy; however, there are not yet any clear cut rules to guide these choices. Further progress at the molecular level of vaccine science will make it possible to produce much safer and more effective mucosal vaccines and vaccine adjuvants in the future.

\section{Author Disclosure Statement}

No competing financial interests exist.

\section{References}

1. Abreu MT. Toll-like receptor signalling in the intestinal epithelium: how bacterial recognition shapes intestinal function. Nat Rev Immunol 2010;10:131-144.

2. Askew D, Harding CV. Antigen processing and CD24 expression determine antigen presentation by splenic CD4+ and CD8+ dendritic cells. Immunology 2008;123:447-455.
3. Blaauboer SM, Gabrielle VD, Jin L. MPYS/STINGmediated TNF-alpha, not type I IFN, is essential for the mucosal adjuvant activity of (3'-5')-cyclic-di-guanosinemonophosphate in vivo. J Immunol 2014;192:492-502.

4. Blaauboer SM, Mansouri S, Tucker HR, et al. The mucosal adjuvant cyclic di-GMP enhances antigen uptake and selectively activates pinocytosis-efficient cells in vivo. eLife 2015;4:e6670.

5. Cai X, Chiu YH, Chen ZJJ. The cGAS-cGAMP-STING pathway of cytosolic DNA sensing and signaling. Mol Cell 2014;54:289-296.

6. Cario E. Toll-like receptors in inflammatory bowel diseases: a decade later. Inflamm Bowel Dis 2010;16:15831597.

7. Carter NJ, Curran MP. Live attenuated influenza vaccine (FluMist(R); Fluenz): a review of its use in the prevention of seasonal influenza in children and adults. Drugs 2011;71:1591-1622.

8. Cerovic V, Houston SA, Scott CL, et al. Intestinal CD103(-) dendritic cells migrate in lymph and prime effector T cells. Mucosal Immunol 2013;6:104-113.

9. Coelingh K, Olajide IR, MacDonald P, et al. Efficacy and effectiveness of live attenuated influenza vaccine in schoolage children. Expert Rev Vaccines 2015;14:1331-1346.

10. Coffman RL, Sher A, Seder RA. Vaccine adjuvants: putting innate immunity to work. Immunity 2010;33:492-503.

11. Coombes JL, Siddiqui KRR, Arancibia-Carcamo CV, et al. A functionally specialized population of mucosal CD103(+) DCs induces Foxp3(+) regulatory T cells via a TGF-beta- and retinoic acid-dependent mechanism. J Exp Med 2007;204:1757-1764.

12. Dalod M, Chelbi R, Malissen B, et al. Dendritic cell maturation: functional specialization through signaling specificity and transcriptional programming. EMBO J 2014;33: 1104-1116.

13. DePaolo RW, Abadie V, Tang F, et al. Co-adjuvant effects of retinoic acid and IL-15 induce inflammatory immunity to dietary antigens. Nature 2011;471:220-U110.

14. Desch AN, Gibbings SL, Clambey ET, et al. Dendritic cell subsets require cis-activation for cytotoxic CD8 T-cell induction. Nat Commun 2014;5:4674.

15. Dudziak D, Kamphorst AO, Heidkamp GF, et al. Differential antigen processing by dendritic cell subsets in vivo. Science 2007;315:107-111.

16. Edelson BT, Kc W, Juang R, et al. Peripheral CD103+ dendritic cells form a unified subset developmentally related to CD8alpha+ conventional dendritic cells. J Exp Med 2010;207:823-836.

17. Farache J, Koren I, Milo I, et al. Luminal bacteria recruit CD103+ dendritic cells into the intestinal epithelium to sample bacterial antigens for presentation. Immunity 2013;38:581-595.

18. Fougeron D, Van Maele L, Songhet $\mathrm{P}$, et al. Indirect Tolllike receptor 5-mediated activation of conventional dendritic cells promotes the mucosal adjuvant activity of flagellin in the respiratory tract. Vaccine 2015;33:3331-3341.

19. Fujimoto K, Karuppuchamy T, Takemura N, et al. A new subset of CD103+CD8alpha+ dendritic cells in the small intestine expresses TLR3, TLR7, and TLR9 and induces Th1 response and CTL activity. J Immunol 2011;186: 6287-6295.

20. Goulopoulou S, McCarthy CG, Webb RC. Toll-like receptors in the vascular system: sensing the dangers within. Pharmacol Rev 2016;68:142-167. 
21. Guilliams M, Ginhoux F, Jakubzick C, et al. Dendritic cells, monocytes and macrophages: a unified nomenclature based on ontogeny. Nat Rev Immunol 2014;14:571-578.

22. Hayashi M, Aoshi T, Haseda Y, et al. Advax, a delta inulin microparticle, potentiates in-built adjuvant property of coadministered vaccines. EBioMedicine 2017;15:127-136.

23. Hayashi M, Aoshi T, Ozasa K, et al. RNA is an adjuvanticity mediator for the lipid-based mucosal adjuvant, endocine. Sci Rep 2016;6:29165.

24. Hildner K, Edelson BT, Purtha WE, et al. Batf3 deficiency reveals a critical role for CD8alpha+ dendritic cells in cytotoxic T cell immunity. Science 2008;322:1097-1100.

25. Kim SH, Jang YS. Antigen targeting to $M$ cells for enhancing the efficacy of mucosal vaccines. Exp Mol Med 2014;46:e85.

26. Koyama S, Aoshi T, Tanimoto $\mathrm{T}$, et al. Plasmacytoid dendritic cells delineate immunogenicity of influenza vaccine subtypes. Sci Transl Med 2010;2:25ra24.

27. Kusakabe $\mathrm{T}$, Ozasa $\mathrm{K}$, Kobari $\mathrm{S}$, et al. Intranasal hydroxypropyl-beta-cyclodextrin-adjuvanted influenza vaccine protects against sub-heterologous virus infection. Vaccine 2016;34:3191-3198.

28. Lewis DJ, Huo Z, Barnett S, et al. Transient facial nerve paralysis (Bell's palsy) following intranasal delivery of a genetically detoxified mutant of Escherichia coli heat labile toxin. PLoS One 2009;4:e6999.

29. Lewis KL, Caton ML, Bogunovic M, et al. Notch2 receptor signaling controls functional differentiation of dendritic cells in the spleen and intestine. Immunity 2011; 35:780-791.

30. Lycke N. Recent progress in mucosal vaccine development: potential and limitations. Nat Rev Immunol 2012;12:592605.

31. Madhun AS, Haaheim LR, Nostbakken JK, et al. Intranasal c-di-GMP-adjuvanted plant-derived H5 influenza vaccine induces multifunctional Th1 CD4+ cells and strong mucosal and systemic antibody responses in mice. Vaccine 2011;29:4973-4982.

32. Maltais AK, Stittelaar KJ, Veldhuis Kroeze EJ, et al. Intranasally administered endocine formulated 2009 pandemic influenza $\mathrm{H} 1 \mathrm{~N} 1$ vaccine induces broad specific antibody responses and confers protection in ferrets. Vaccine 2014;32:3307-3315.

33. Maroof A, Yorgensen YM, Li Y, et al. Intranasal vaccination promotes detrimental Th17-mediated immunity against influenza infection. PLoS Pathog 2014;10: e1003875.

34. Mashayekhi M, Sandau MM, Dunay IR, et al. CD8alpha(+) dendritic cells are the critical source of interleukin-12 that controls acute infection by Toxoplasma gondii tachyzoites. Immunity 2011;35:249-259.

35. Mazzini E, Massimiliano L, Penna G, et al. Oral tolerance can be established via gap junction transfer of fed antigens from CX3CR1(+) macrophages to CD103(+) dendritic cells. Immunity 2014;40:248-261.

36. McDole JR, Wheeler LW, McDonald KG, et al. Goblet cells deliver luminal antigen to CD103+ dendritic cells in the small intestine. Nature 2012;483:345-349.

37. Mildner A, Jung S. Development and function of dendritic cell subsets. Immunity 2014;40:642-656.

38. Murphy TL, Grajales-Reyes GE, Wu X, et al. Transcriptional control of dendritic cell development. Annu Rev Immunol 2016;34:93-119.
39. Mutsch M, Zhou W, Rhodes P, et al. Use of the inactivated intranasal influenza vaccine and the risk of Bell's palsy in Switzerland. N Engl J Med 2004;350:896-903.

40. Neutra MR, Kozlowski PA. Mucosal vaccines: the promise and the challenge. Nat Rev Immunol 2006;6:148158.

41. Nochi T, Yuki Y, Takahashi H, et al. Nanogel antigenic protein-delivery system for adjuvant-free intranasal vaccines. Nat Mater 2010;9:572-578.

42. Nohynek H, Jokinen J, Partinen M, et al. AS03 adjuvanted AH1N1 vaccine associated with an abrupt increase in the incidence of childhood narcolepsy in Finland. PLoS One 2012;7:e33536.

43. Onishi M, Ozasa K, Kobiyama K, et al. Hydroxypropylbeta-cyclodextrin spikes local inflammation that induces $\mathrm{Th} 2$ cell and $\mathrm{T}$ follicular helper cell responses to the coadministered antigen. J Immunol 2015;194:2673-2682.

44. Partinen M, Kornum BR, Plazzi G, et al. Narcolepsy as an autoimmune disease: the role of $\mathrm{H} 1 \mathrm{~N} 1$ infection and vaccination. Lancet Neurol 2014;13:600-613.

45. Piccinini AM, Midwood KS. DAMPening inflammation by modulating TLR signalling. Mediators Inflamm 2010; 672395.

46. Reed SG, Orr MT, Fox CB. Key roles of adjuvants in modern vaccines. Nat Med 2013;19:1597-1608.

47. Roush SW, Murphy TV. Vaccine-Preventable Disease Table Working Group. Historical comparisons of morbidity and mortality for vaccine-preventable diseases in the United States. JAMA 2007;298:2155-2163.

48. Satpathy AT, Briseno CG, Lee JS, et al. Notch2-dependent classical dendritic cells orchestrate intestinal immunity to attaching-and-effacing bacterial pathogens. Nat Immunol 2013;14:937-948.

49. Schlitzer A, Ginhoux F. Organization of the mouse and human DC network. Curr Opin Immunol 2014;26:90-99.

50. Schlitzer A, McGovern N, Ginhoux F. Dendritic cells and monocyte-derived cells: two complementary and integrated functional systems. Semin Cell Dev Biol 2015;41:9-22.

51. Scott CL, Bain CC, Wright PB, et al. CCR2(+)CD103(-) intestinal dendritic cells develop from DC-committed precursors and induce interleukin-17 production by $\mathrm{T}$ cells. Mucosal Immunol 2015;8:327-339.

52. Stary G, Olive A, Radovic-Moreno AF, et al. VACCINES. A mucosal vaccine against Chlamydia trachomatis generates two waves of protective memory $\mathrm{T}$ cells. Science 2015;348:aaa8205.

53. Suzuki H, Watari A, Hashimoto E, et al. C-terminal Clostridium perfringens enterotoxin-mediated antigen delivery for nasal pneumococcal vaccine. PLoS One 2015;10.

54. Takeuchi O, Akira S. Pattern recognition receptors and inflammation. Cell 2010;140:805-820.

55. Tussiwand R, Everts B, Grajales-Reyes GE, et al. Klf4 expression in conventional dendritic cells is required for T helper 2 cell responses. Immunity 2015;42:916-928.

56. Uematsu S, Fujimoto K, Jang MH, et al. Regulation of humoral and cellular gut immunity by lamina propria dendritic cells expressing Toll-like receptor 5. Nat Immunol 2008;9:769-776.

57. Van Maele L, Fougeron D, Janot L, et al. Airway structural cells regulate TLR5-mediated mucosal adjuvant activity. Mucosal Immunol 2014;7:489-500.

58. Vesikari T, Knuf M, Wutzler P, et al. Oil-in-water emulsion adjuvant with influenza vaccine in young children. $\mathrm{N}$ Engl J Med 2011;365:1406-1416. 
59. Wang $\mathrm{S}$, Liu $\mathrm{H}$, Zhang $\mathrm{X}$, et al. Intranasal and oral vaccination with protein-based antigens: advantages, challenges and formulation strategies. Protein Cell 2015;6: 480-503.

60. Woodrow KA, Bennett KM, Lo DD. Mucosal vaccine design and delivery. Annu Rev Biomed Eng 2012;14:17-46.

61. $\mathrm{Wu} \mathrm{J}$, Chen $\mathrm{ZJ}$. Innate immune sensing and signaling of cytosolic nucleic acids. Annu Rev Immunol 2014;32:461488.

62. Yang Y, Torchinsky MB, Gobert M, et al. Focused specificity of intestinal $\mathrm{T}(\mathrm{H}) 17$ cells towards commensal bacterial antigens. Nature 2014;510:152-156.

63. Ye LL, Zeng RY, Bai Y, et al. Efficient mucosal vaccination mediated by the neonatal Fc receptor. Nat Biotechnol 2011;29:158-163.

64. Yin Y, Qin T, Wang X, et al. CpG DNA assists the whole inactivated H9N2 influenza virus in crossing the intestinal epithelial barriers via transepithelial uptake of dendritic cell dendrites. Mucosal Immunol 2015;8:799-814.

65. Yu M, Yang Y, Zhu C, et al. Advances in the transepithelial transport of nanoparticles. Drug Discov Today 2016;21:1155-1161.

Address correspondence to:

Dr. Taiki Aoshi

Vaccine Dynamics Project

BIKEN Innovative Vaccine Research Alliance Laboratories Research Institute for Microbial Disease

Osaka University

3-1 Yamadaoka, Suita

Osaka 565-0871

Japan

E-mail: aoshi@biken.osaka-u.ac.jp 